\title{
Review of: "Relationships between urbanization and CO2 emissions in China: An empirical analysis of population migration"
}

Chi Wei Su

Potential competing interests: The author(s) declared that no potential competing interests exist.

I think this paper is well structured and written. I think it's worth publishing in this journal. 